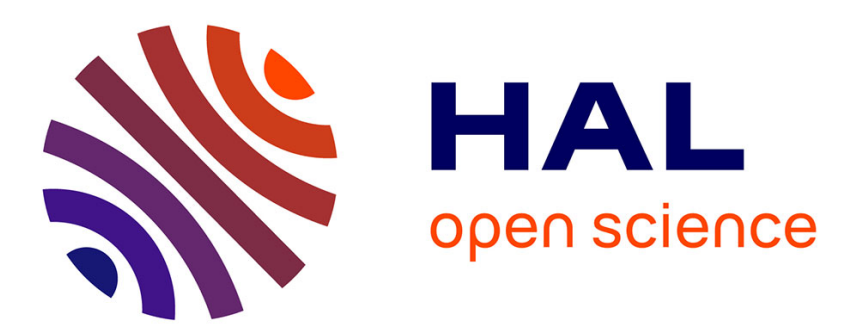

\title{
The Guided Bilateral Filter: When the Joint/Cross Bilateral Filter Becomes Robust
}

Laurent Caraffa, Jean Philippe Tarel, Pierre Charbonnier

\section{To cite this version:}

Laurent Caraffa, Jean Philippe Tarel, Pierre Charbonnier. The Guided Bilateral Filter: When the Joint/Cross Bilateral Filter Becomes Robust. IEEE Transactions on Image Processing, 2015, 24 (4), pp 1199-1208. 10.1109/TIP.2015.2389617 . hal-01215837

\section{HAL Id: hal-01215837 \\ https://hal.science/hal-01215837}

Submitted on 15 Oct 2015

HAL is a multi-disciplinary open access archive for the deposit and dissemination of scientific research documents, whether they are published or not. The documents may come from teaching and research institutions in France or abroad, or from public or private research centers.
L'archive ouverte pluridisciplinaire HAL, est destinée au dépôt et à la diffusion de documents scientifiques de niveau recherche, publiés ou non, émanant des établissements d'enseignement et de recherche français ou étrangers, des laboratoires publics ou privés. 
123 


\title{
The Guided Bilateral Filter: When the Joint/Cross Bilateral Filter Becomes Robust
}

\author{
Laurent Caraffa, Jean-Philippe Tarel*, Member, IEEE, Pierre Charbonnier
}

\begin{abstract}
The bilateral filter and its variants such as the Joint/Cross bilateral filter are well known edge-preserving image smoothing tools used in many applications. The reason of this success is its simple definition and the possibility of many adaptations. The bilateral filter is known to be related to robust estimation. This link is lost by the ad hoc introduction of the guide image in the Joint/Cross bilateral filter. We here propose a new way to derive the Joint/Cross bilateral filter as a particular case of a more generic filter which we name the Guided bilateral filter. This new filter is iterative, generic, inherits the robustness properties of the Robust bilateral filter and uses a guide image. The link with robust estimation allows us to relate the filter parameters with the statistics of input images. A scheme based on Graduated Non Convexity is proposed, which allows converging to an interesting local minimum even when the cost function is non-convex. With this scheme, the Guided bilateral filter can handle non-Gaussian noise on the image to be filtered. A complementary scheme is proposed to handle also non-Gaussian noise on the guide image even if both are strongly correlated. This allows the Guided bilateral filter to handle situations with more noise than the Joint/Cross bilateral filter can work with and leads to high Peak Signal to Noise Ratio $P S N R$ values as shown experimentally.
\end{abstract}

Index Terms-image filtering, image smoothing, Bilateral filter, Joint bilateral filter, Cross bilateral filter, Dual bilateral filter.

\section{INTRODUCTION}

The bilateral filter [1] is a well known edge-preserving image smoothing tool. The key idea of the bilateral filter consists in introducing a photometric weight into the standard Gaussian filter. The effect of this weight is to cancel spatial interactions between pixels with an important intensity difference. Thanks to its properties, the bilateral filter is now used in many different applications, for instance: sharpness enhancement [2], upsampling [3], depth map refinement [4], image editing [5] and fog removal [6].

A good summary on bilateral filter, interpretations, improvements and extensions is given in [7]. In our opinion, there are five important points to recall. First, the bilateral filter is connected to the robust estimation of the intensity average in a neighborhood [8]. Second, the staircase effect which may be observed in the results can be canceled by using linear fitting rather constant fitting [9]. Third, the bilateral filter can

Copyright (c) 2015 IEEE. Personal use of this material is permitted. However, permission to use this material for any other purposes must be obtained from the IEEE by sending a request to pubs-permissions@ieee.org.

L. Caraffa and J.-P. Tarel are with the Paris-Est University, IFSTTAR (COSYS/LEPSIS), 14-20 Boulevard Newton, F-77420 Champs-sur-Marne, France, e-mail: laurent.caraffa@ifsttar.fr and jean-philippe.tarel@ifsttar.fr

P. Charbonnier is with the Cerema (ERA 27 IFSTTAR), 11 Rue Jean Mentelin, BP 9, F-67200 Strasbourg, France, e-mail: pierre.charbonnier@cerema.fr be extended as the adaptive bilateral filter where the scale parameter in the photometric weight is selected using the intensities of the neighbor pixels [2]. Fourth, there are several ways to improve the computation time of the bilateral filter, for instance using a grid [10] or using distributive histograms [11]. Fifth, in some situations, information about the structure of the target image is available under the form of a similar image, called the guide image. The latter may be used to define a guide weight which may be introduced, either in place of the photometric weight (leading to the Joint/Cross bilateral filter [12], [13]) or in conjunction with it (Dual bilateral filter [14]). Note that the guide image has also been used alone in [6], but in a framework different from bilateral filtering. While the improvements proposed in the second to fourth points only slightly impact the interpretation of the bilateral filter in terms of robust estimation, the ad hoc introduction of the guide in the joint/cross bilateral filter calls for a new statistical interpretation.

In this paper, we first recall in Section II the link between the bilateral filter and robust estimation, but we also show, experimentally, that neither the bilateral filter nor its iterative version are really robust. In contrast, applying a full Iterative Reweighted Least Squares (IRLS) iteration leads to the socalled Robust bilateral filter [15]. In the same spirit, we propose in Section III to connect the Joint/Cross bilateral and Dual bilateral filters to robust estimation. This leads us to extend these filters into a novel one, iterative and robust, the Guided bilateral filter. All the filters we use or introduce here are listed in Table I with their main features recalled.

TABLE I

CHARACTERISTICS OF THE FILTERS USED OR INTRODUCED IN THE PAPER.

\begin{tabular}{|c||c|c|c|c|c|c|}
\hline Filter name & $\begin{array}{c}\text { Spatial } \\
\text { weight }\end{array}$ & $\begin{array}{c}\text { Photo. } \\
\text { weight }\end{array}$ & $\begin{array}{c}\text { Guide } \\
\text { weight }\end{array}$ & Iterative & Robust & $\begin{array}{c}\text { Ref. } \\
\text { or eq. }\end{array}$ \\
\hline \hline Gaussian & Yes & No & No & one step & No & \\
Bilateral & Yes & Yes & No & one step & No & {$[1],(1)$} \\
Iterated bilateral & Yes & Yes & No & several steps & No & $(1)$ \\
Robust bilateral & Yes & Yes & No & IRLS steps & Yes & {$[15],(3)$} \\
Joint/Cross bilateral & Yes & No & Yes & one step & No & {$[13],[12]$} \\
Dual bilateral & No & Yes & Yes & one step & No & {$[14]$} \\
Guided & No & No & Yes & one step & No & {$[6]$} \\
Guided bilateral & Yes & Yes & Yes & IRLS steps & Yes & $(8)$ \\
\hline
\end{tabular}

In Section IV, a convergence scheme based on Graduated Non Convexity is proposed for the Guided bilateral filter. Then, in Section V, we show how the input images statistics can be used to set the filter parameters and we experiment with the proposed filter under different kinds of added noise. These results compare advantageously with results obtained 
with bilateral, Joint/Cross bilateral, Dual bilateral, and Guided filters. Finally, in Section VI, the interest of the proposed filter is illustrated on three applications: flash/no-flash filtering, depth map refinement and fog removal.

\section{BILATERAL FILTER}

The bilateral filter proposed in [1] is an extension of the linear smoothing of images where a photometric weight $w_{p}$ is introduced as a factor of the spatial weight $w_{s}$. From the original image $E$, the result of the bilateral filter is the image $F$ given by:

$$
F(x)=\frac{\sum_{t \in S_{m}} w_{s}(\|t\|) w_{p}(E(x)-E(x+t)) E(x+t)}{\sum_{t \in S_{m}} w_{s}(\|t\|) w_{p}(E(x)-E(x+t))}
$$

where $S_{m}$ is, typically, a square window $[-m, m] \times[-m, m]$. $w_{s}$ is a symmetrical, decreasing function of the distance $\|t\|$ from the center of $S_{m}$. Usually, $w_{p}$ is also an even and decreasing function of the intensity.

\section{A. Robust bilateral filter}

According to [8], the image $F$ resulting from the bilateral filter can be interpreted as the first step of the minimization, with respect to $F(x)$, of the local cost:

$$
\sum_{t \in S_{m}} w_{s}(\|t\|) \phi\left((F(x)-E(x+t))^{2}\right)
$$

When $w_{s}(\|t\|)=1$, the minimizer $F(x)$ of the cost is the robust average of the values $E(x+t), t \in S_{m}$. In such a case, $\phi$ characterizes the average noise model on the intensities in the neighborhood $S_{m}$. By canceling the derivative of the sum in (2) with respect to $F(x)$, we have:

$\sum_{t \in S_{m}} w_{s}(\|t\|) \phi^{\prime}\left((F(x)-E(x+t))^{2}\right)(F(x)-E(x+t))=0$

After rearranging the terms and fixing non-linear terms to their previously computed value, the well-known IRLS algorithm is obtained, which iteratively minimizes (2):

$$
F_{k+1}(x)=\frac{\sum_{t \in S_{m}} w_{s}(\|t\|) \phi^{\prime}\left(\left(F_{k}(x)-E(x+t)\right)^{2}\right) E(x+t)}{\sum_{t \in S_{m}} w_{s}(\|t\|) \phi^{\prime}\left(\left(F_{k}(x)-E(x+t)\right)^{2}\right)}
$$

Derivation and proofs of convergence of the IRLS iterations may be found for instance in [16], [17]. By comparing (1) with (3), we see that the bilateral filter is the first step of the IRLS algorithm when $w_{p}(u)=\phi^{\prime}\left(u^{2}\right)$ and when it is initialized with the original image (i.e. $F_{0}(x)=E(x)$ ). We name Robust bilateral filter, the IRLS iterations until convergence.

\section{B. Robustness assessment}

On Fig. 1, an original image, chosen for its difficulty (presence of thin image structures) is shown, along with the image to be processed, where a Gaussian noise ( $\operatorname{std} s=5)$ and a Salt and Pepper (10\%) noise were added. The result with the
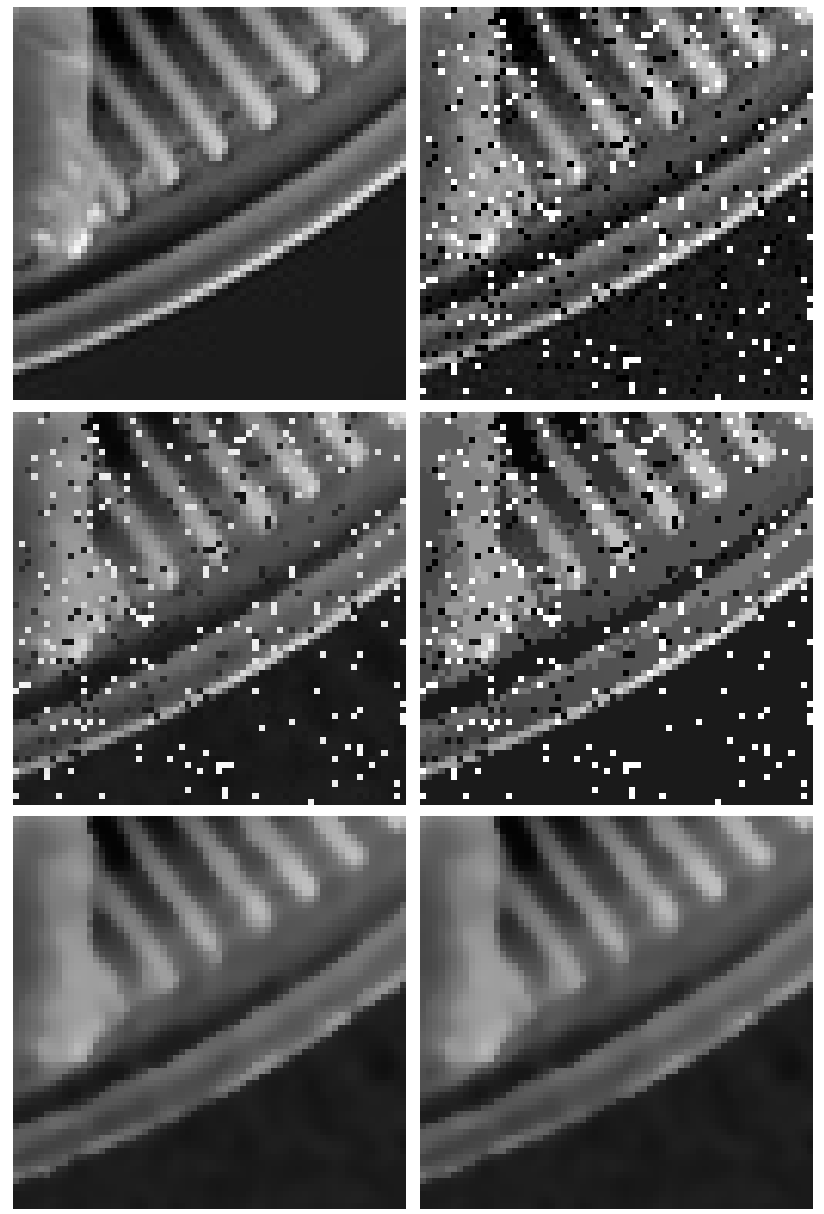

Fig. 1. Comparison between bilateral filter, iterated bilateral filter and Robust bilateral filter. First row: left, the original image and right, the noisy image $(P S N R=14.5 d B)$. Second row: result with the bilateral filter after 1 and 100 iterations $\left(\alpha_{p}=-1, s_{p}=70, P S N R=15.5 d B\right.$ and $\alpha_{p}=-1, s_{p}=$ $5, P S N R=14.6 d B$, respectively). Third row: result of the Robust bilateral filter with 10 and 100 iterations $\left(\alpha_{p}=0.5, s_{p}=5, P S N R=28.9 d B\right.$ and $28.8 d B$, respectively).

bilateral filter and after 100 iterations of the bilateral filter is shown on the second row. Despite the fact that it is related to robust estimation as previously explained, the bilateral filter is not robust to non-Gaussian noise in the original image. The iterative application of the bilateral filter is not robust to outliers either.

On the contrary, the Robust bilateral filter introduced in the previous section is robust as illustrated by the results with 10 and 100 iterations, shown on the third row of Fig. 1. It is important to distinguish between the iterated bilateral filter and the Robust bilateral filter. Indeed, in the Robust bilateral filter (3), $F_{k}$ is compared to the fixed values of $E$, while, in the iterative application of the bilateral filter, $F_{k}$ is compared to the value obtained from the previous filter application.

Due to its derivation, the Robust bilateral filter is robust to outliers in the original image, provided that the noise model is suited to the input and that the correct convergence scheme is used. 

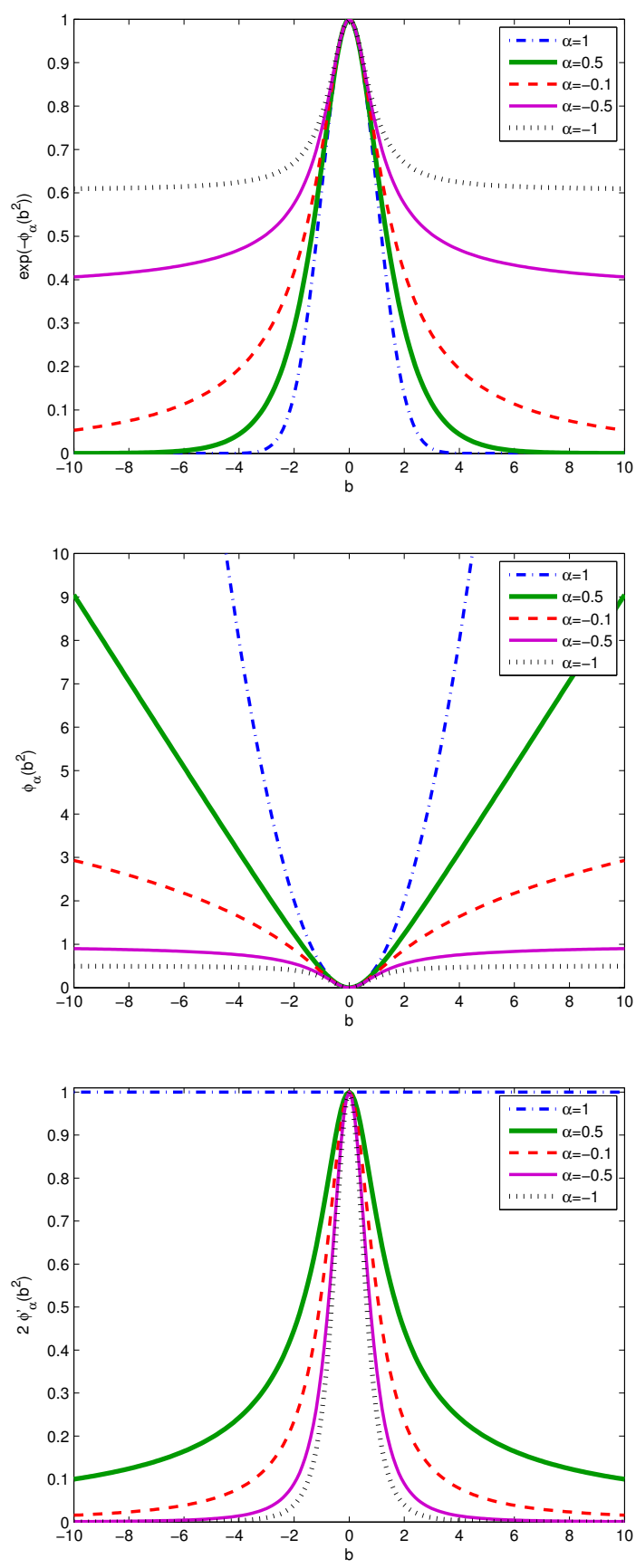

Fig. 2. Plots of Smooth Exponential Family (SEF) functions. From top to bottom, for different values of $\alpha$ : the $\operatorname{pdf} \exp \left(-\phi_{\alpha}\left(b^{2}\right)\right)$, the error function $\phi_{\alpha}\left(b^{2}\right)$, and the corresponding weight $\phi_{\alpha}^{\prime}\left(b^{2}\right)$.

\section{Noise model}

For modeling different kinds of centered additive noise, the following parametric probability density function (pdf) will be used:

$$
p_{\alpha, s}(b) \propto \frac{1}{s} e^{-\phi_{\alpha}\left(\left(\frac{b}{s}\right)^{2}\right)}
$$

where $\phi_{\alpha}(t)=\frac{1}{2 \alpha}\left((1+t)^{\alpha}-1\right)$. This is the so-called Smooth Exponential Family (SEF) introduced in [18], [15]. The shape of the pdf is tuned by two parameters: $s$, the scale of the noise and $\alpha$, which allows a continuous transition between different kinds of useful pdfs, see Fig. 2. In particular, the Gaussian pdf is obtained for $\alpha=1$. The Laplace pdf is approximated with $\alpha=0.5$, and Geman \& McClure's pdf [19] is obtained for $\alpha=-1$. The Cauchy/T-student pdfs are obtained when $\alpha \rightarrow 0$ as a limit case by integration of $\phi^{\prime}$ [15]. The robustness of the Maximum Likelihood Estimator (MLE) derived from the SEF family is studied in [15], where it is shown that the robustness is increasing when $\alpha$ is decreasing from 1 . The maximum $50 \%$ robustness is achieved for $\alpha \leq 0$.

The cost (2) can be derived as a MLE from the probability of the residual $b=F(x)-E(x+t)$ :

$$
p(b) \propto\left(\frac{1}{s} e^{-\phi_{\alpha}\left(\left(\frac{b}{s}\right)^{2}\right)}\right)^{q_{t}}
$$

where $q_{t}$ is an indicator of the similarity of the noiseless intensities at pixels locations $x$ and $(x+t)$. The value of this similarity indicator $q_{t}$ is assumed to lie in the interval $[0,1]$. The value $q_{t}=1$, indicates that the random variables at locations $x$ and $(x+t)$ have the same center values. In that case, $p(b)$, the pdf of $b$ in (5), is within the SEF model (4). A value of $q_{t}$ close to zero indicates that the random variables at locations $x$ and $(x+t)$ have very different center values. In that case, $p(b)$ becomes closer to the uniform distribution, so the contribution of this pair to the overall likelihood is neutralized. This pair is not informative. In the bilateral filter, the similarity indicator $q_{t}$ is assumed to be known and is set to the spatial weight $w_{s}(\|t\|)$.

\section{Graduated Non Convexity}

As explained in [15], the convergence to a good local minimum requires a careful convergence scheme, based on the Graduated Non Convexity (GNC) [20], [15] strategy. When the SEF family $\phi_{\alpha_{p}, s_{p}}$ is used, the GNC consists in:

- applying first iterations using $\alpha_{p}=1$ or $\alpha_{p}=0.5$, to enforce convexity of the minimized cost,

- performing a sequence of fits with a stepwise decreasing $\alpha_{p}$ in continuation, i.e. each time using the current output image as an initial value for the next filtering step. This is performed until the target value of $\alpha_{p}$ is achieved.

For values $\alpha_{p} \geq 0.5$, the cost function (2) is always convex as the sum of convex functions. Such an example is shown in the third row of Fig. 1. For values $\alpha_{p}<0.5$, it is not necessarily convex, so several local minima may exist. Alternatively, the cost (2) can be minimized exhaustively (by enumeration of gray level values) to achieve the global minimum. Using the GNC, we observed in practice that a minimum very close to the global one is obtained at a lower cost. Indeed the number of iterations to achieve convergence is reduced by a factor between 5 and 30 .

In our experimentations, we observed that, during the first steps of the GNC, outliers are attenuated in the current result but image details are also lost. During the next ten steps of the GNC, the details are progressively restored, but without reintroducing outliers. However, one may observe that fine structures are not perfectly restored. We will see in Sec. III that using a guide image may be very beneficial from this point of view. 


\section{E. Linear fitting}

Due to the modeling by a constant in (2), the iterated bilateral and Robust bilateral filters are subject to staircase effects in regions with smooth gradient of gray level or colors. Following [9], the staircase effect can be removed by using a local linear fitting in the neighborhood of each pixel. The iteration consists now in an IRLS on the parameters of the local 2D plane:

$$
\begin{array}{r}
\left(\begin{array}{c}
H_{k+1}(x) \\
F_{k+1}(x)
\end{array}\right)=\left(R+\sum_{t \in S_{m}} w_{s}(\|t\|) w_{p, k}\left(\begin{array}{l}
t \\
1
\end{array}\right)\left(\begin{array}{l}
t \\
1
\end{array}\right)^{T}\right)^{-1} \\
\sum_{t \in S_{m}} w_{s}(\|t\|) w_{p, k} E(x+t)\left(\begin{array}{l}
t \\
1
\end{array}\right)
\end{array}
$$

with $w_{p, k}=\phi^{\prime}\left(\left(H_{k}^{T}(x) t+F_{k}(x)-E(x+t)\right)^{2}\right)$, where $R$ is a diagonal matrix chosen to enforce the positivity of the inverse matrix in (6), and with $T$ for transpose. $H_{k}(x)$ is the slope of the 2D plane at iteration $k$, and the filter result is the intercept $F_{k}(x)$ of the 2D plane. As in the previous section, the convergence towards an interesting local minimum requires using the GNC approach.

\section{GUIDED BILATERAL FILTER}

In several applications, another image of the scene, taken in different conditions or with a different spectral band, is available in addition to the input image. This image is named guide image. Note that its photometry is usually distorted or different, see for instance Fig. 9. The Joint/Cross bilateral filter was introduced in an ad hoc way simultaneously in [13], [12], from the bilateral filter, by substituting the original image $E$ with the guide image $G$ in the expression of the photometric weight $w_{p}$, in (1). The guide image $G$ indicates where similar pixels are located in each neighborhood. Due to this substitution, the link with robust estimation is lost. To restore this link, we here propose to keep $w_{p}$ and to introduce the guide image $G$ into a third weight $w_{g}$, in cost (2). This extra weight, we name guide weight, was introduced inside the bilateral filter equation to obtain the Dual bilateral filter [14] but not in the cost function as we do. In our opinion, and contrary to [13], [12], this weight $w_{g}$ is a similarity indicator, like the spatial weight, and not a photometric weight. Indeed, the guide image can be seen as a label image which describes the structure of similar pixels in each neighborhood. This leads us to introduce a new filter which we name the Guided bilateral filter, whose output is the value $F(x)$ achieved at the minimum of the following cost:

$$
\sum_{t \in S_{m}} \underbrace{w_{s}(\|t\|) w_{g}(G(x)-G(x+t))}_{q_{t}} \phi\left((F(x)-E(x+t))^{2}\right)
$$

where $w_{s}$ is still the spatial weight, $w_{g}$ is the guide weight from the guide image, $q_{t}=w_{s} w_{g}$ is the new similarity indicator and $\phi$ characterizes the photometric noise model. Like the Robust bilateral filter, the Guided bilateral filter is iterative and derives as a MLE from (5) but with a different form for $q_{t}$. It thus can be written as:

$$
F_{k+1}(x)=\frac{\sum_{t \in S_{m}} q_{t} \phi^{\prime}\left(\left(F_{k}(x)-E(x+t)\right)^{2}\right) E(x+t)}{\sum_{t \in S_{m}} q_{t} \underbrace{\phi^{\prime}\left(\left(F_{k}(x)-E(x+t)\right)^{2}\right)}_{w_{p}\left(F_{k}(x)-E(x+t)\right)}}
$$

with: $q_{t}=w_{s}(\|t\|) w_{g}(G(x)-G(x+t))$.

The Robust bilateral filter is the particular case of the Guided bilateral filter when $w_{g}=1$. The Joint/Cross bilateral filter is the first iteration of (8) when $w_{p}=1$. The Dual bilateral filter is the first iteration of the Guided bilateral filter, when $w_{s}=1$. Contrary to the Cross/Joint bilateral and Dual bilateral filters, the Guided bilateral filter is robust to nonGaussian noise on the original image if the weight $w_{p}$ or equivalently if $\phi^{\prime}$ are adequately chosen w.r.t. the image noise. A summary of the main features of these different filters is shown in Tab. I.

The Guided bilateral filter is very flexible thanks to its three weights. In practice, the spatial weight $w_{s}$ can be set to 1 when a good quality guide $G$ is provided. Indeed, the guide weight $w_{g}$ is usually more informative about the similarity between pixels than the spatial weight $w_{s}$. When the guide is not of good quality, a Gaussian function can be used for the spatial weight, as we do in the experiments: $w_{s}(\|t\|)=e^{-\frac{1}{2}\left(\frac{\|t\|}{s_{s}}\right)^{2}}$ where $s_{s}$ is the scale of the spatial weight. In the experiments, we also found convenient to set the function $w_{g}(c)$ to $p_{\alpha_{g}, s_{g}}(c): w_{g}(c)=e^{-\phi_{\alpha_{g}}\left(\left(\frac{c}{s_{g}}\right)^{2}\right)}$ where $\alpha_{g}$ and $s_{g}$ are the two parameters of the guide weight.

The photometric weight $w_{p}$ is related to the nature of the noise on the image $E$, as for the Robust bilateral filter. We now suppose that the function $w_{p}(b)$ is chosen as SEF weight $w_{p}(b)=\phi_{\alpha_{p}}^{\prime}\left(\left(\frac{b}{s_{p}}\right)^{2}\right)$, and thus parameterized by two values $\alpha_{p}$ and $s_{p}$. This assumption is important to derive the convergence scheme using GNC. Notice that when the noise is Gaussian, this implies $\alpha_{p}=1$ and thus $w_{p}(b)$ equals one. In case of color or multi-components images, the squared difference which is in the photometric and guide weights is substituted by a sum over the components of squared differences. As in Sec. II-E, a local fitting by a linear model can be used rather than a fitting by a constant model in $w_{p}$ to cancel the staircase effect.

As in Sec. II-B, when the function $\phi$ is not convex, the convergence towards an interesting local minimum requires the GNC approach, as detailed in the next section.

The Guided bilateral filter is applied to the same original image as in Fig. 1 where Gaussian (std $s=5)$ plus SP (10\%) noise is added. The guide image is, in this example, a gamma transform of the original image without added noise. On the second row of Fig. 3 are shown the results of the Joint/Cross bilateral filter and after 100 iterations (with $s_{s}=1, \alpha_{g}=$ $\left.1, s_{g}=5\right)$. In the first case, the outliers are not completely removed: the Joint/Cross bilateral filter is not really a robust filter. With 100 iterations, the outliers are better removed, but small details are also removed due to the iterations, which involve spatial convolutions of increasing scale. On the third row are shown the results of the Guided bilateral filter (with $w_{s}=1, \alpha_{g}=0, \alpha_{p}=-1, s_{g}=s_{p}=5$ ) when a local fitting 

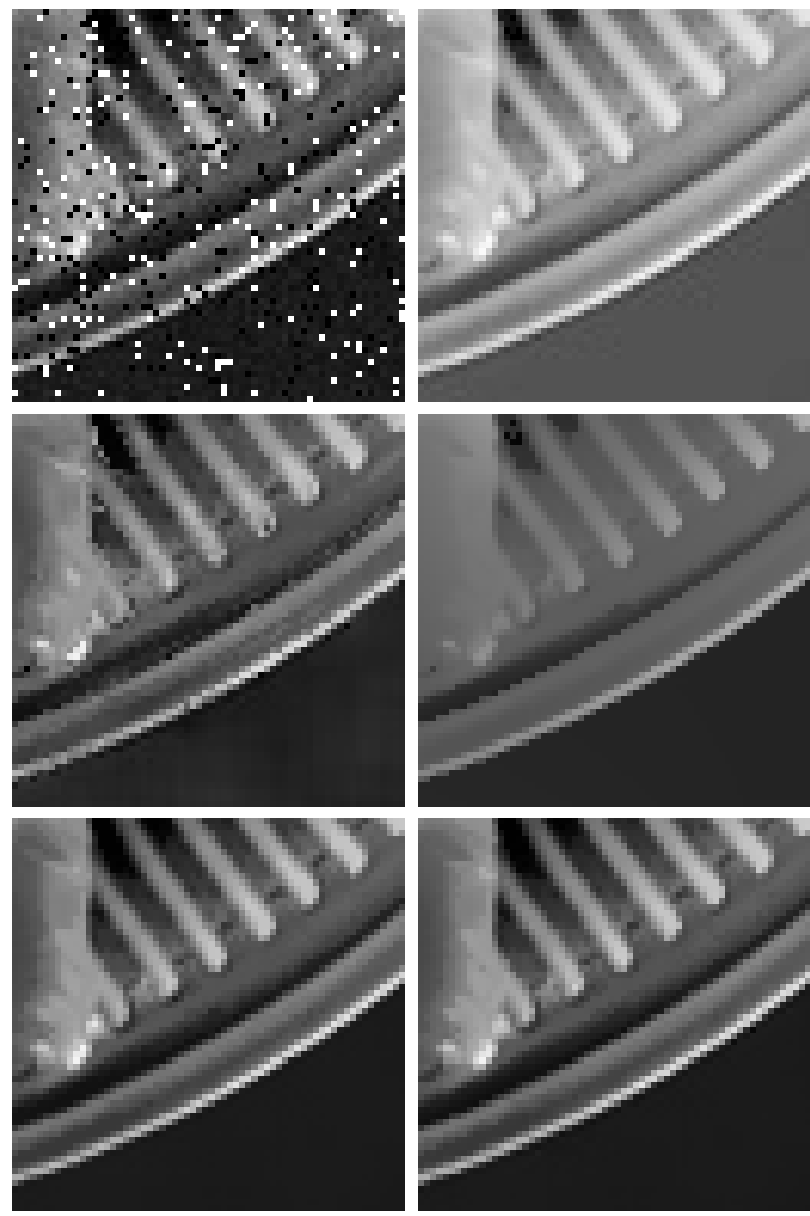

Fig. 3. Comparison between the Joint/Cross bilateral filter and the Guided bilateral filter, in case of a noiseless guide. First row: left, the noisy image $E$ to be processed ( $P S N R=14.5 \mathrm{~dB}$ ) and right, the guide image $G$, which in this example is a noiseless gamma-transformed version of the original image $(P S N R=13.1 d B)$. Second row: result of the Joint/Cross bilateral filter after 1 and 100 iterations $(P S N R=27.4 d B$ and $P S N R=20.7 d B)$. Third row: result of the Guided bilateral filter with constant $(P S N R=39.6 \mathrm{~dB})$ and with linear local fitting $(P S N R=38.5 \mathrm{~dB})$.

is used with constant and linear models. The results obtained with the Guided bilateral filter are drastically superior in terms of PSNR compared to the results of the Joint/Cross bilateral filter, iterated or not. This can be explained by the fact that no spatial convolution of increasing scale is involved.

\section{CONVERGENCE SCHEME}

We now provide a scheme for a convergence towards an interesting local minimum when the guide image is free of noise. Then, we consider the case where a noise is also added to the guide image, and even more difficult, when both observed noises are the same.

\section{A. Small noise on the guide}

Without noise on the guide, the scheme we found to converge quickly towards an interesting local minimum in 8 iterations consists in the following heuristic:

- set the power $\alpha_{g}$ and the scale $s_{g}$ as a function of the intensities of the details to be kept, select the power $\alpha_{p}$ and the scale $s_{p}$ as a function of the noise in the image $E$, and assume the spatial weight to be one;

- apply one step of the Guided bilateral filter with $w_{p}=1$ to the noisy image;

- on the result, apply one iteration of the Guided bilateral filter using the previous set of parameters but with a temporary $\alpha_{p}=0.5$;

- if the chosen value of $\alpha_{p}$ is lower than zero, then apply one extra iteration of the Guided bilateral filter using a temporary $\alpha_{p}=0$;

- finally, apply extra iterations of the Guided bilateral filter using the value $\alpha_{p}$ chosen in the first step until the maximum number of 8 iterations is achieved.

We ran experiments with added synthetic noise. For constant model and parameters $\left(\alpha_{g}=0.5, \alpha_{p}=-1\right.$, and $\left.s_{g}=s_{p}=5\right)$, the energy obtained using the GNC scheme is, on 10 images with Gaussian plus SP noise, on average 1.0022 times the energy of the global solution. The achieved energy using GNC and exhaustive enumeration are thus very close. On these images, the $P S N R$ obtained by exhaustive enumeration is $37.2 \pm 0.5 d B$. Surprisingly with the GNC, the obtained $P S N R$ is higher: $39.2 \pm 0.2 d B$, with a smaller variance. Indeed, when the number of iterations increases, the $P S N R$ first increases rapidly then decreases slowly, after 10 iterations. The smaller variance can be explained by the use of the same initialization during the GNC, which biases the result towards the local minimum closest to the initialization. Similar conclusions are obtained when a Cauchy noise is added.

\section{B. Strong noise on the guide}

On the original image of Fig. 1, we studied the cases where Gaussian (std $s=5, P S N R=13.1 d B)$ or SP $(10 \%$, $P S N R=11.1 \mathrm{~dB}$ ) noise is added both to the guide and to the image. In both cases, we observed that difficulties arise at pixels where the observed noise on the image $E$ and on the guide $G$ are similar in value. This leads us to experiment with the most difficult situation where the same observed SP noise is added onto the original image and onto the guide, as shown in the first row of Fig. 4. In the last row of Fig. 4, we see that the SP noise is not removed by the Guided bilateral filter (with $\alpha_{g}=0, \alpha_{p}=-1, s_{g}=s_{p}=5$ ). The Guided bilateral filter is not robust to noise when it is correlated between the input image and the guide. The solution is to pre-process the guide by filtering it using for instance the Robust bilateral filter (with $s_{s}=1, \alpha_{p}=-1, s_{p}=5$ ). After this pre-processing of the guide, the previous GNC scheme is applied on the input image, with one difference: $w_{s}$ is no longer one, but it is set to a Gaussian function, with a small scale such as $s_{s}=1.0$ or $s_{s}=1.5$. The spatial weight is used to account for the lower reliability of the guide compared to the noiseless case.

On the image of Fig. 4, a rather good $P S N R=29.0 \mathrm{~dB}$ value is obtained using the proposed pre-processing. In comparison, with the Robust bilateral filtering with the same parameters, the $P S N R$ is equal to $27.4 d B$. Compared to $P S N R=29.0 d B$, the results obtained using the Joint/Cross bilateral filter are of lower $P S N R$ in both cases, without $\left(w_{s}=1, \alpha_{g}=0, s_{g}=70\right)$ and with pre-processing $\left(s_{s}=1.5\right.$, $\left.\alpha_{g}=0, s_{g}=70\right)$. 

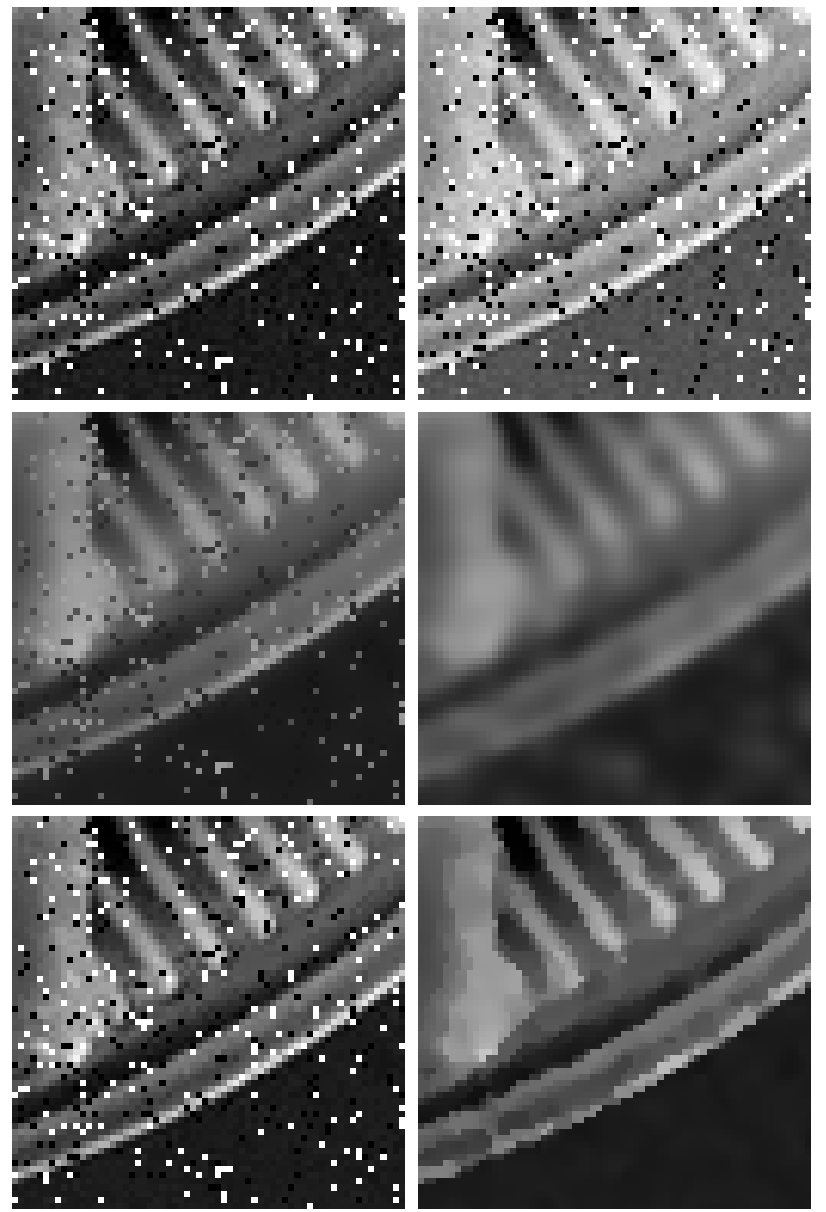

Fig. 4. Comparison between the Joint/Cross bilateral filter and the Guided bilateral filter, in the presence of a noisy guide. First row: left, the image $E$ to be processed and right, guide image $G$ with the same noise. Second row: result of the Joint/Cross bilateral filter without (left, $P S N R=23.3 d B$ ) and with (right, $P S N R=21.5 d B$ ) filtering of the guide. Third row: result of the Guided bilateral filter without $(P S N R=14.5 d B)$ and with $(P S N R=$ $29.0 d B$ ) filtering of the guide.

\section{TESTS}

\section{A. Influence of parameters}

The parameters of the Guided filter are: the power $\alpha_{g}$ and the scale $s_{g}$ of the guide weight $w_{g}$, the power $\alpha_{p}$ and the scale $s_{p}$ of the photometric weight $w_{p}$, and possibly the scale $s_{s}$ of the spatial weight $w_{s}$, if the latter is assumed Gaussian.

On the original image, named Hudson Diatom, from which the image of Fig. 1 was extracted, we studied the performance changes as a function of the two parameters $\alpha_{p} \in$ $[-0.5, \cdots, 1.0]$ and $s_{p} \in[1, \cdots, 20]$, depending on the kind of added noise. We use three different noises: Gaussian $(\alpha=1)$, Laplace $(\alpha=0.5)$ and Cauchy $(\alpha=0)$, with the same scale parameter, $s=10$. As shown on Fig. 5, the variations of the $P S N R$ with respect to $\alpha_{p}$ and $s_{p}$ are relatively smooth. The best $P S N R$ value is achieved for $\alpha_{p}=1$ in the Gaussian noise case, as expected. When a Laplace noise is added, the best $P S N R$ s are achieved for $\alpha_{p}=0.5$, for two values of the scale: $s_{p}=1$ and $s_{p}$ around 8 . When a Cauchy noise is added, the best PSNRs are achieved when $s_{p}=6$ for $\alpha_{p}=0$ and also when $s_{p}=1$ for $\alpha_{p}=0.5$. These two maxima can be
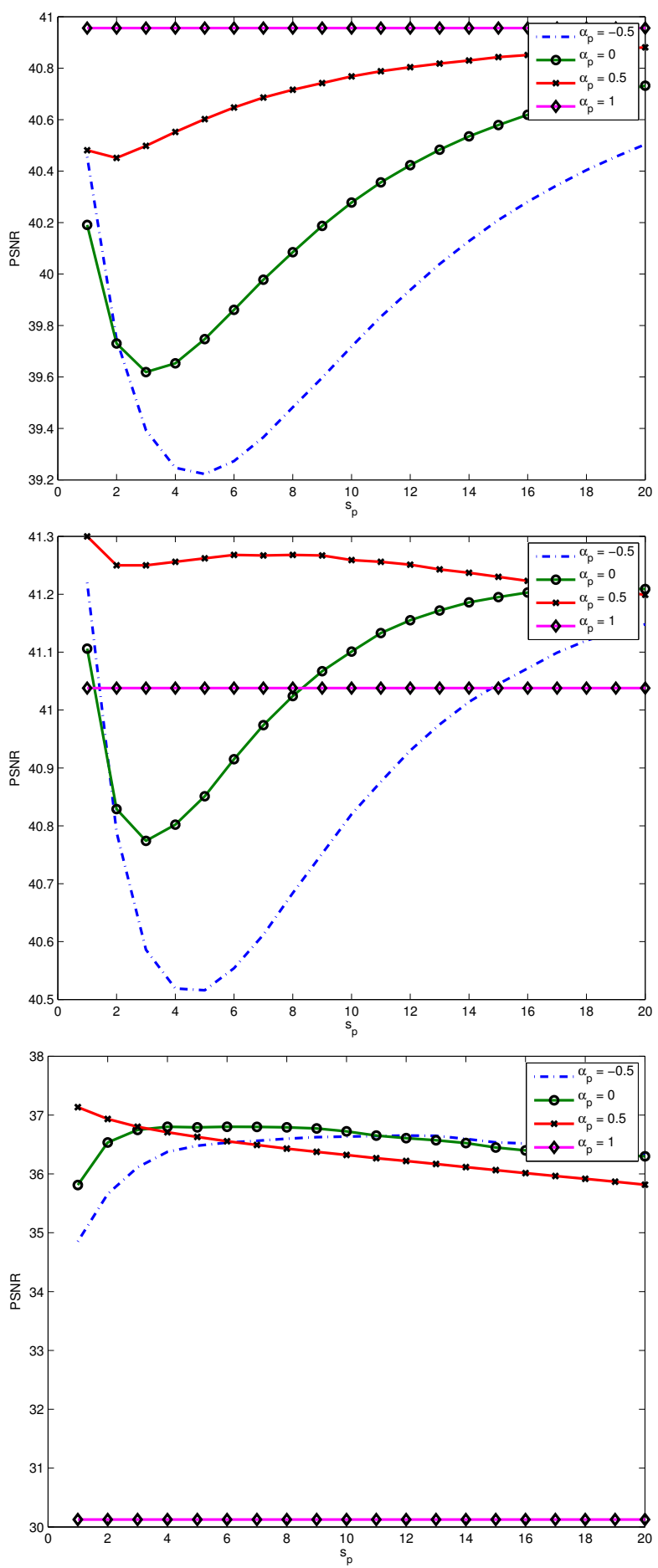

Fig. 5. Variations of the $P S N R$ for different parameter values of the photometric weight. From top to bottom, three different kinds of noise are added to the image Hudson Diatom: Gaussian $(\alpha=1)$, Laplace $(\alpha=0.5)$ and Cauchy $(\alpha=0)$ with same scale parameter $(s=10)$. PSNR variations are relatively smooth.

explained by the fact that the intensity is thresholded between 0 and 255.

In Fig. 6, the $P S N R$ variations are displayed for three different images when the added noise is a Cauchy noise. In the first column, $P S N R$ variations are displayed w.r.t. $\alpha_{p}$ and 

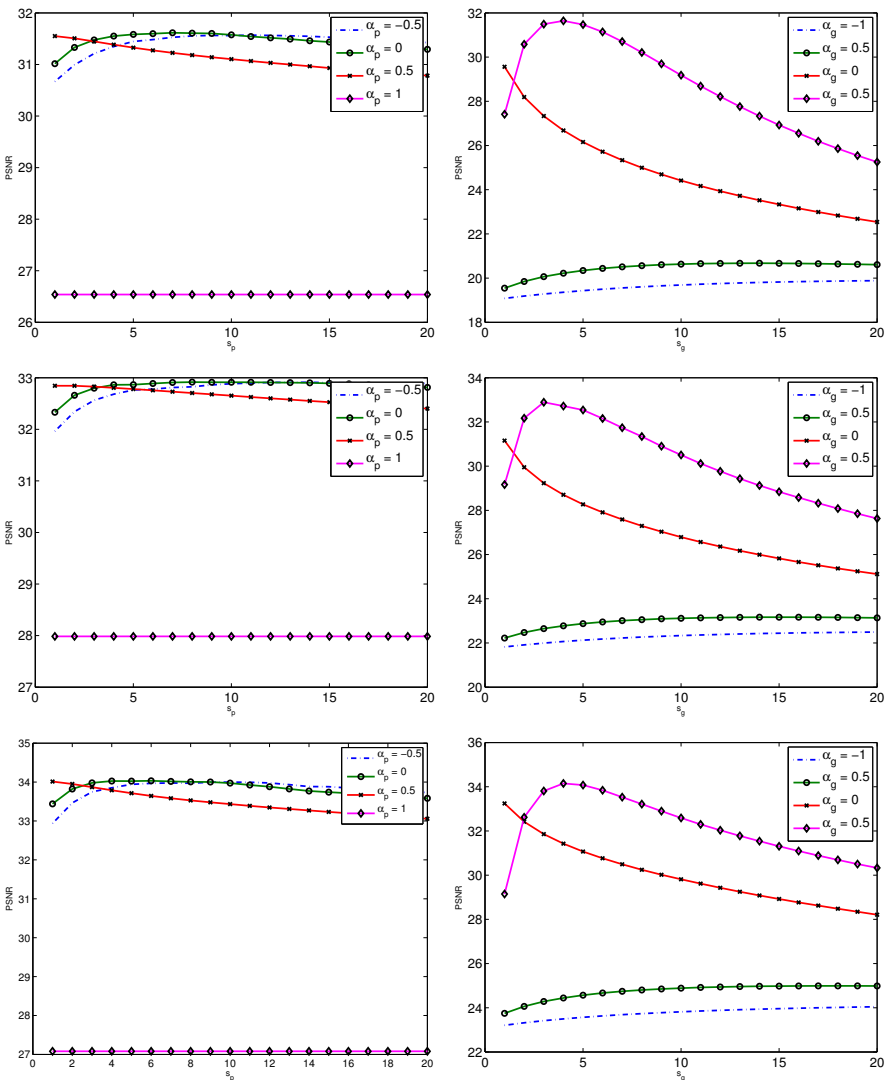

Fig. 6. Variations of the $P S N R$ for different parameter values of the photometric weight (first column) and of the guide weight (second column). From top to bottom, three different images are used: Baboon, Fishing boat and Peppers. A crop of these images can be see on Fig. 8. The added noise is of Cauchy type $(\alpha=0)$ with scale parameter $s=10$. PSNR variations are relatively smooth and similar from one image to another.

$s_{p}$ and in the second column, w.r.t. $\alpha_{g}$ and $s_{g}$. The shape of the $P S N R$ curves are rather similar from one image to another. This illustrates the fact that the parameter values should be set as a function of the image noise and not as a function of the image content. These $P S N R$ variations are relatively smooth w.r.t. the four parameters of the photometric and guide weights. This illustrates that the Guided bilateral filter does not need very accurate parameter tuning to provide almost optimal performance.

\section{B. Parameter values from image statistics}

The statistical interpretation of the weights in the Robust bilateral filter, described in the end of Sec.II-A, is interesting since it suggests an approximate way to select the product of the spatial and photometric weight. Indeed, if we forget about the noise on the window center, the pdf $p(b)$ in (5) can be observed from the residuals $E(x)-E(x+t)$, for every $\|t\|$, from the input image. In Fig. 7, we show in red the residual histogram for three additive noises (Gaussian, Laplace and Cauchy with scale $s=10$ ), on the same image. Each histogram is fitted within the SEF. The estimated parameters provide interesting values to set the parameters of the weights of the Robust bilateral filter. In Fig. 7, the fit of each residual histogram is shown in black. The fitted parameter $(\alpha, s)$
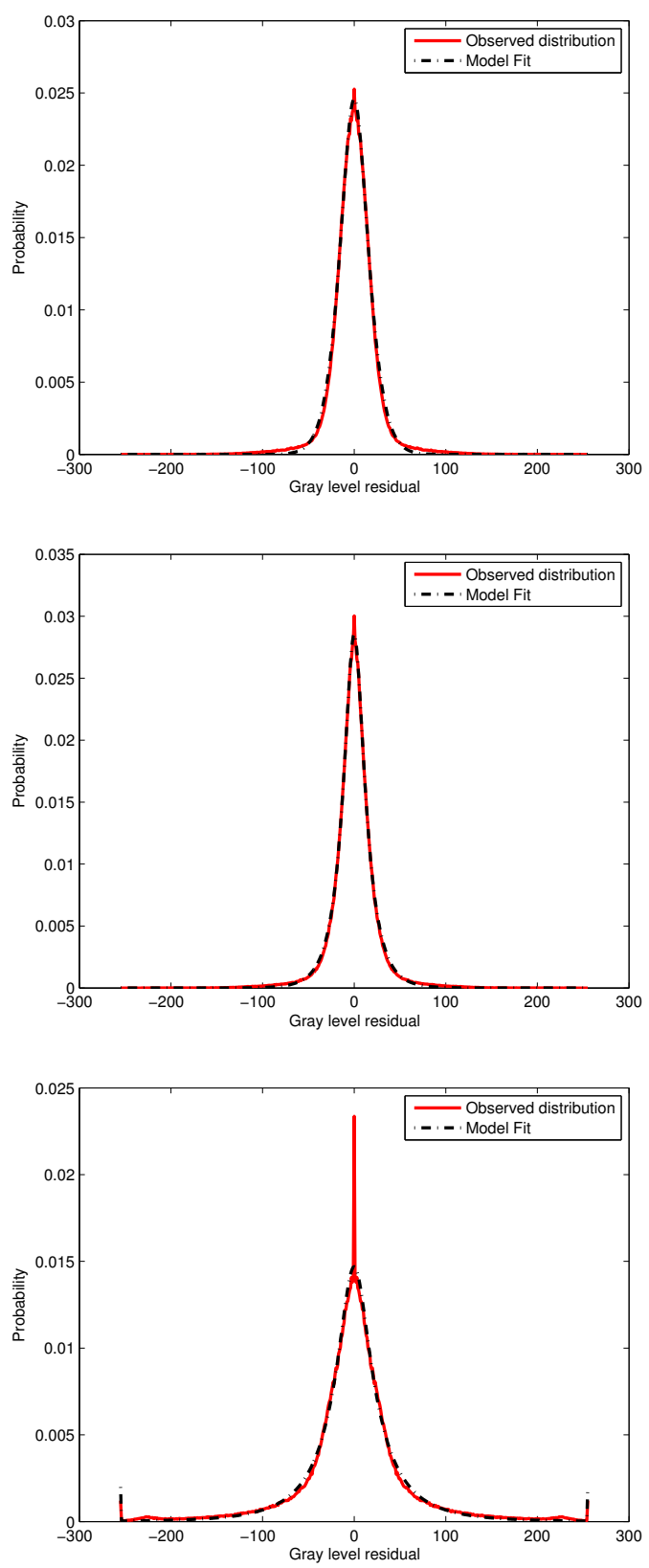

Fig. 7. Fits of the noise observed on the images. In red, the obtained histogram of intensity residuals when different kinds of noise are added (from top to bottom: Gaussian, Laplace and Cauchy with scale $s=10$ ). In black, the fit in the SEF.

values are $(0.63,13.6),(0.38,9.6),(0.23,15.4)$ respectively for Gaussian, Laplace and Cauchy noise. Using these values for the restoration of 10 different images, the resulting average $P S N R$ are $40.8 d B, 41.2 d B$ and $36.3 d B$ respectively. Such values almost correspond to the maximum $P S N R$ achieved in Fig. $5(40.9 d B, 41.3 d B$ and $37.0 d B$ respectively). The loss in $P S N R$ is thus in average $0.8 \%$ compared to the use of the true parameters. 

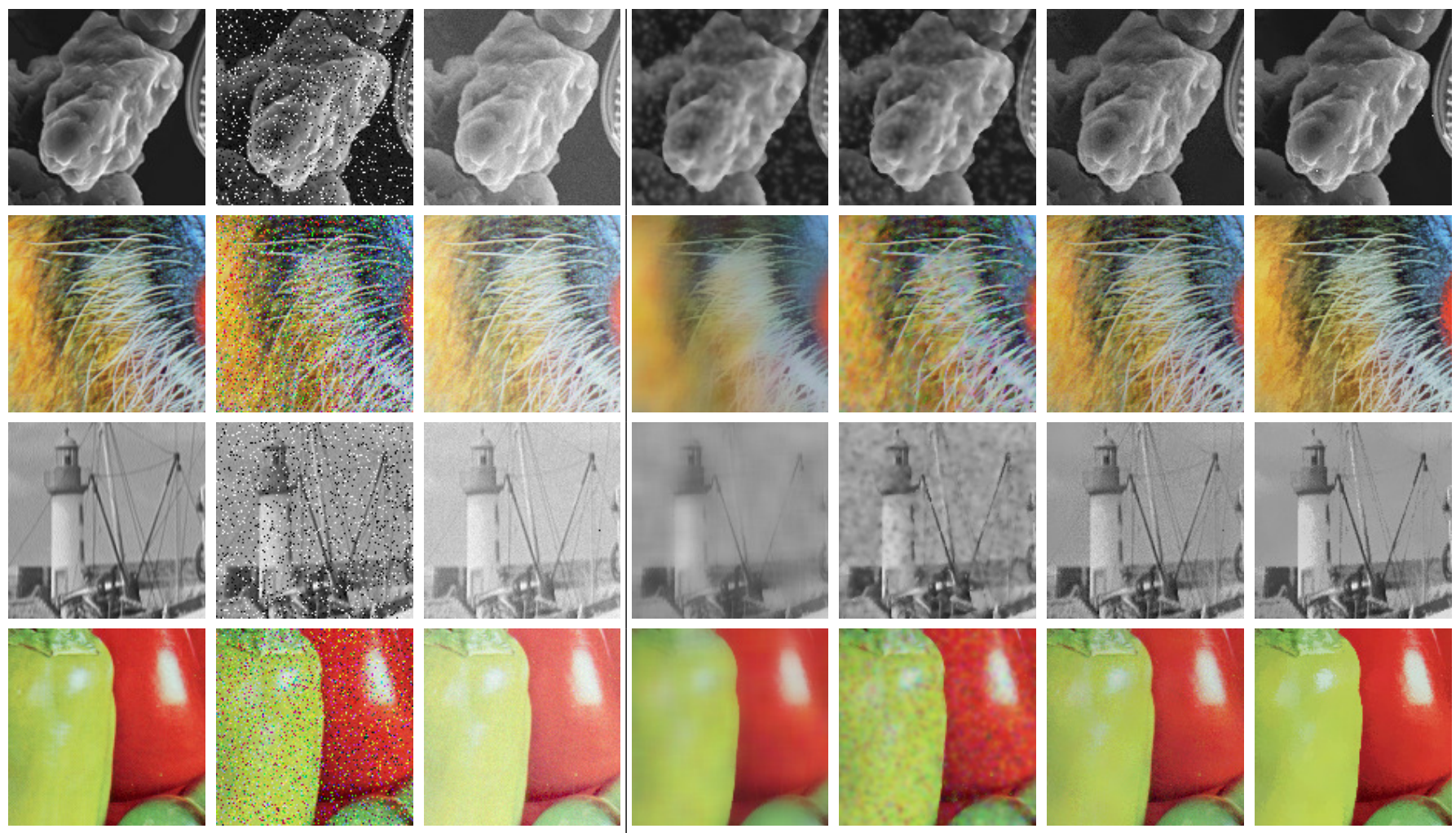

Fig. 8. Comparison on four image crops. From left to right, column-wise: the original image, the image with Gaussian (std $s=5)$ plus SP (10\%) noise, the guide (gamma transform of the original, noiseless image with $\gamma=1.4$ ), result of the Joint/Cross Bilateral filter, Dual Bilateral filter [14], Guided filter [6] and Guided Bilateral filter. For each filter, parameter values are selected as the ones that achieve the best $P S N R$ in average on the four images.

\section{Comparison}

The interesting performances of the Guided bilateral filter are confirmed on several images in presence of SP (10\%) noise on the input image and in the presence of Gaussian $(s=5)$ noise on the guide, see crops of Fig. 8. On this figure, the Guided bilateral filter is compared to the Joint/Cross bilateral filter and to the Guided filter introduced in [6]. The best performance in terms of $P S N R$ over a range of parameter values is displayed in Tab. II. Notice that the Guided bilateral filter always outperforms the three other filters, by several $d B$ s.

\section{TABLE II}

BEST PSNR (IN $d B$ ) OVER A RANGE OF PARAMETER VALUES FOR THE FOUR IMAGES OF FIG. 8 WITH JOINT/CROSS BILATERAL FILTER, DUAL BILATERAL FILTER [14], GUIDED FILTER [6] AND GUIDED BILATERAL FILTER.

\begin{tabular}{|c||c|c|c|c|}
\hline $\begin{array}{c}\text { Image } \\
\text { name }\end{array}$ & $\begin{array}{c}\text { Joint/cross } \\
\text { bilateral } \\
\text { filter }\end{array}$ & $\begin{array}{c}\text { Dual } \\
\text { bilateral } \\
\text { filter }\end{array}$ & $\begin{array}{c}\text { Guided } \\
\text { filter }\end{array}$ & $\begin{array}{c}\text { Guided } \\
\text { bilateral } \\
\text { filter }\end{array}$ \\
\hline \hline Hudson diatom & 24.9 & 25.0 & 28.3 & 35.3 \\
Baboon & 13.4 & 24.1 & 28.7 & 31.6 \\
Fishing boat & 14.0 & 26.1 & 29.9 & 32.9 \\
Peppers & 14.2 & 25.5 & 28.6 & 34.03 \\
\hline
\end{tabular}

\section{Applications}

To illustrate the practical interest of the proposed Guided bilateral filter, we now provide a few examples of its application to flash/no-flash filtering, depth map refinement and fog removal.

\section{A. Flash/no-flash filtering}
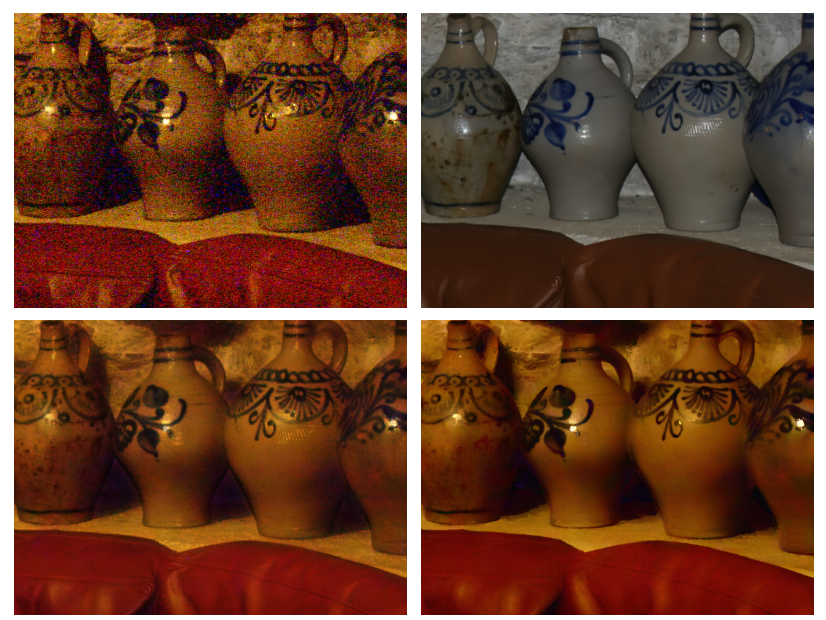

Fig. 9. Example of flash/no flash filtering. First row: original images without and with flash. Second row: left, result obtained following [13] using the bilateral filter on the flash image and the Cross/Joint bilateral filter on the no-flash image; right, result obtained using the Robust bilateral filter on the flash image and the Guided bilateral filter on the no-flash image.

In Fig. 9, we used the original flash/no-flash images of [13] and the same method to remove the noise in the no-flash image with the flash image. To obtain the leftmost image on the second row, the flash picture is first filtered using the bilateral filter. Then the no-flash picture is filtered using the Joint/Cross bilateral filter with the smooth flash image as a guide. Finally, 
the details of the flash image are introduced by multiplying the previous result with the ratio of the flash image over the smooth flash image. The rightmost image on the second row of Fig. 9 is obtained by using the Robust bilateral filter with parameters $w_{s}=1, \alpha_{g}=1, s_{g}=1, \alpha_{p}=-1, s_{p}=3$ instead of a bilateral filter, and using the Guided bilateral filter with parameters $w_{s}=1, \alpha_{g}=0, s_{g}=10, \alpha_{p}=-1$, $s_{p}=40$ rather than a Joint/Cross bilateral filter. Due to the robustness of both filters, the result is smoother, and noise is entirely removed. Moreover, the specularities of the flash image are less transferred onto the no-flash image compared to the previous result.

\section{B. Depth map refinement}
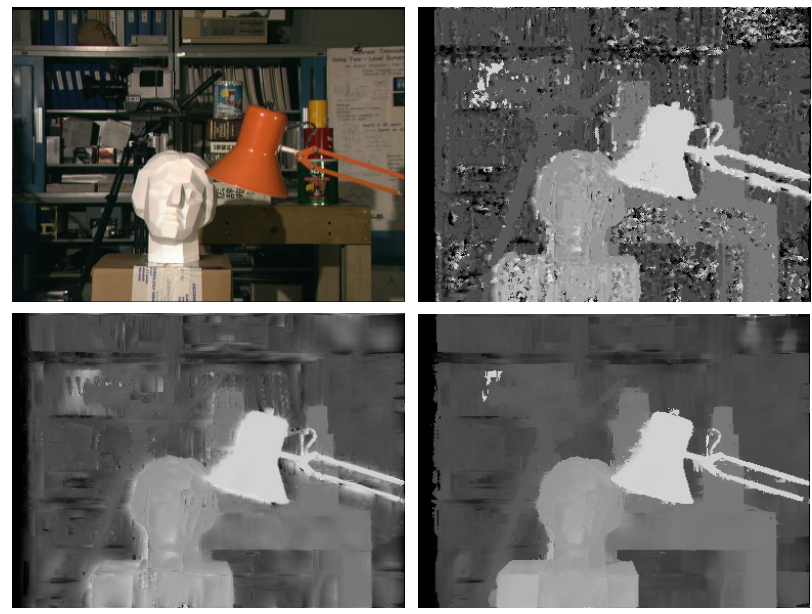

Fig. 10. Example of depth map refinement. First row: left, original image of the stereo pair; right, disparity map obtained using a dense stereo reconstruction algorithm. Second row: the refined disparity maps after filtering by the Joint/Cross bilateral filter (left) and after the Guided bilateral filter (right).

The Joint/Cross bilateral filter was used in [4] for depth map refinement and interpolation using one of the original images of the stereo pair as a guide. In Fig. 10, a disparity map is obtained using a belief propagation algorithm on a Markov random field model of the problem which was voluntarily stopped before total convergence to save processing time. Even if numerous outliers are present in the original image, the refinement based on the Guided bilateral filter achieves interesting results. To obtain these results, the GNC approach was performed with parameters $w_{s}=1, \alpha_{g}=0, s_{g}=10$, $\alpha_{p}=-1, s_{p}=40$. The result obtained using the Joint/Cross bilateral filter is less convincing, as shown on Fig. 10.

\section{Single image fog removal}

In this example, following [21], fog removal is performed in two steps on a single image: first, atmospheric veil is estimated from the color saturation (maximum over color channels for each pixel) after median filtering; second, the veil is used to remove the fog by reversing Koschmieder's law, which models fog effects. Between these two steps, the atmospheric veil can be refined by applying the Guided bilateral filter on the veil using the original color image as a guide. This extra step allows better removing the fog between objects with thin shapes such as leaves, as shown on Fig. 11. The parameters of the Guided bilateral filter are $w_{s}=1, \alpha_{g}=0.5, s_{g}=30$, $\alpha_{p}=1, s_{p}=10$ in this example.

\section{CONCLUSION}

In this paper, we have reviewed the derivation of the bilateral filter and several of its most efficient variants from the point of view of robust statistics. In particular, we experimentally shown that an IRLS implementation (called Robust bilateral filter) is the only one that fully guarantees robustness in the presence of noise and outliers. We proposed a probabilistic interpretation of the weights that may be used to unify the derivation of the filters and naturally leads to the definition of a more generic filter, which we name the Guided bilateral filter. The inputs of this filter are two images: the image to be processed and the guide image. The Guided bilateral filter requires four parameters if the guide is of good quality or else five parameters. We provided insight and recommendations for setting these parameters depending on the input data. The proposed filter is iterative, hence we provide a heuristic scheme, based on the Graduated Non Convexity approach which helps converging towards a good local minimum of the cost function, in a reduced number of iterations. Experimentally, in terms of psnr, the Guided bilateral filter outperforms the three other filters which take into account a guide image. The proposed filter is robust to outliers in the image to be processed but not to outliers in the guide image. To overcome this limitation, simply we suggest to pre-process the guide, using the Robust bilateral filter, for instance. We show the interest of the proposed filter for flash/no-flash filtering, depth map refinement and fog removal, but many other applications could profit from its use, such as visible/infrared filtering, sharpness enhancement, upsampling, image editing, gray level image coloring, image recoloring, or depth of field effect [22].

\section{REFERENCES}

[1] C. Tomasi and R. Manduchi, "Bilateral filtering for gray and color images," in ICCV, 1998, pp. 839-846.

[2] B. Y. Zhang and J. P. Allebach, "Adaptive bilateral filter for sharpness enhancement and noise removal," IEEE Trans. Image Processing, vol. 17, no. 5, pp. 664-678, May 2008.

[3] J. Kopf, M. F. Cohen, D. Lischinski, and M. Uyttendaele, "Joint bilateral upsampling," ACM Trans. on Graphics, vol. 26, no. 3, July 2007.

[4] Q. X. Yang, R. G. Yang, J. Davis, and D. Nister, "Spatial-depth super resolution for range images," in $C V P R, 2007$, pp. 1-8.

[5] E. A. Khan, E. Reinhard, R. Fleming, and H. Buelthoff, "Image-based material editing," ACM Trans. on Graphics, vol. 25, no. 3, pp. 654-663, July 2006.

[6] K. He, J. Sun, and X. Tang, "Guided image filtering," in European Conference on Computer Vision (ECCV'10), Hersonissos, Crete, Greece, 2010, pp. 1-14.

[7] S. Paris, P. Kornprobst, J. Tumblin, and F. Durand, "Bilateral filtering: Theory and applications," Foundations and Trends@ in Computer Graphics and Vision, vol. 4, no. 1, pp. 1-75, 2009.

[8] M. Elad, "On the origin of the bilateral filter and ways to improve it," IEEE Trans. Image Processing, vol. 11, no. 10, pp. 1141-1151, Oct. 2002.

[9] A. Buades, B. Coll, and J. M. Morel, "The staircasing effect in neighborhood filters and its solution," IEEE Trans. Image Processing, vol. 15, no. 6, pp. 1499-1505, Jun. 2006.

[10] F. Durand and S. Paris, "A fast approximation of the bilateral filter using a signal processing approach," in ECCV, 2006, pp. IV: 568-580. 

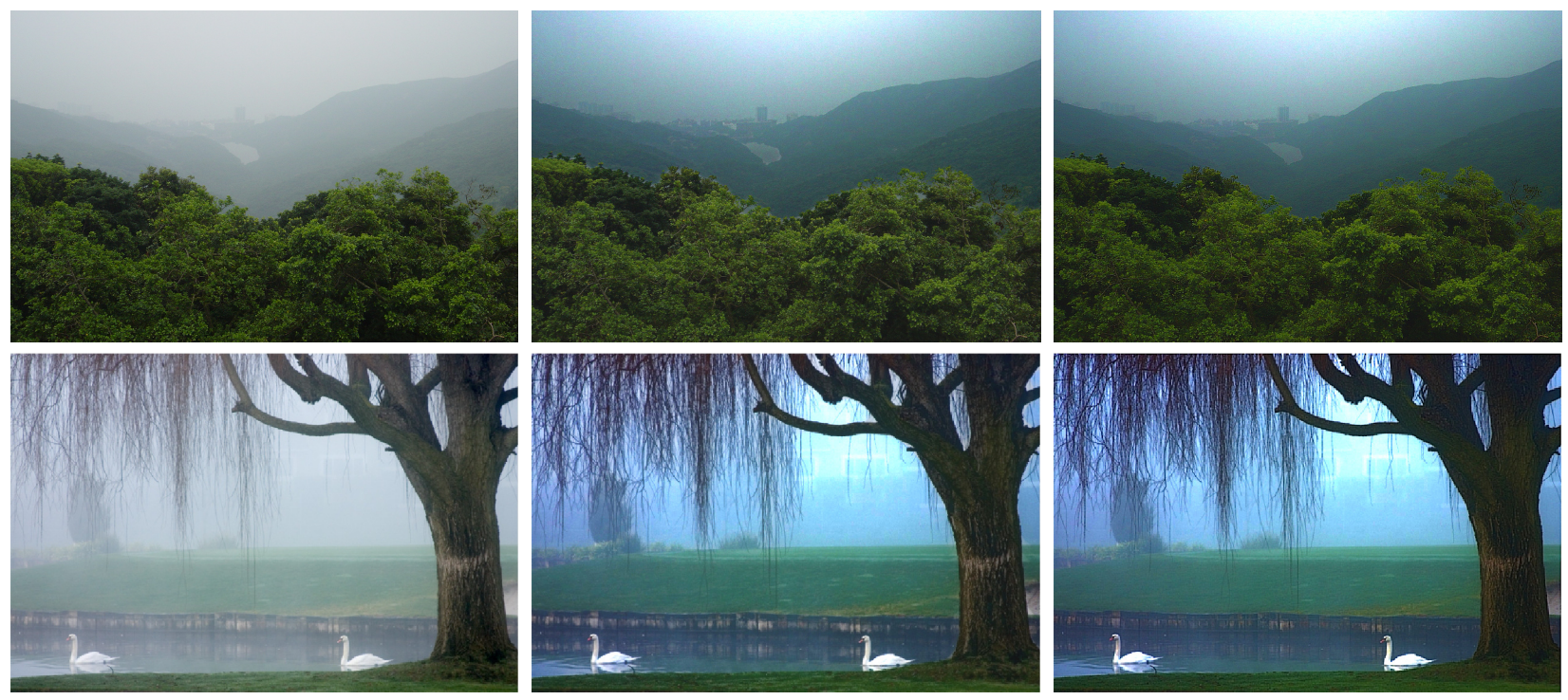

Fig. 11. Example of single image defogging. First column: original foggy images. Second column: fog removal using the fast median filter [21]. Third column: fog removal using the Guided bilateral filter. Notice how halos produced by the median filter are removed with the Guided bilateral filter.

[11] B. Weiss, "Fast median and bilateral filtering," ACM Trans. on Graphics, vol. 25, no. 3, pp. 519-526, July 2006

[12] E. Eisemann and F. Durand, "Flash photography enhancement via intrinsic relighting," ACM Trans. on Graphics, vol. 23, no. 3, pp. 673678, Aug. 2004

[13] G. Petschnigg, R. Szeliski, M. Agrawala, M. Cohen, H. Hoppe, and K. Toyama, "Digital photography with flash and no-flash image pairs," ACM Trans. on Graphics, vol. 23, no. 3, pp. 664-672, Aug. 2004.

[14] E. P. Bennett, J. L. Mason, and L. McMillan, "Multispectral bilateral video fusion," IEEE Trans. Image Processing, vol. 16, no. 5, pp. 11851194, May 2007.

[15] S.-S. Ieng, J.-P. Tarel, and P. Charbonnier, "Modeling non-gaussian noise for robust image analysis," in Proceedings of International Conference on Computer Vision Theory and Applications (VISAPP'07), Barcelona, Spain, 2007, pp. 183-190.

[16] J.-P. Tarel, S.-S. Ieng, and P. Charbonnier, "A constrained-optimization based half-quadratic algorithm for robustly fitting sets of linearly parametrized curves," Advances in Data Analysis and Classification, vol. 2, no. 3, pp. 227-239, 2008.

[17] P. Charbonnier, L. Blanc-Féraud, G. Aubert, and M. Barlaud, "Deterministic edge-preserving regularization in computed imaging," IEEE Trans. Image Processing, vol. 6, no. 2, pp. 298-311, 1997.

[18] J.-P. Tarel, S.-S. Ieng, and P. Charbonnier, "Using robust estimation algorithms for tracking explicit curves," in $E C C V$, vol. I, Copenhagen, Denmark, 2002, pp. 492-507.

[19] S. Geman and D. McClure, "Bayesian image analysis: an application to single photon emission tomography," Proc. Statistical Computational Section, Amer. Statistical Assoc., pp. 12-18, 1985.

[20] A. Blake and A. Zisserman, Visual Reconstruction. Cambridge, MA: MIT Press, 1987.

[21] J.-P. Tarel and N. Hautière, "Fast visibility restoration from a single color or gray level image," in ICCV, Kyoto, Japan, 2009, pp. 2201-2208.

[22] E. S. L. Gastal and M. M. Oliveira, "Domain transform for edge-aware image and video processing," ACM Trans. Graph., vol. 30, no. 4, pp. 69:1-69:12, Jul. 2011.

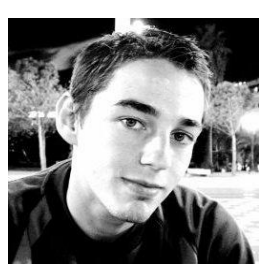

Laurent Caraffa received a M.S. degree in Computer Vision and Image Processing from the University of Nice Sophia-Antipolis in 2010. He received his $\mathrm{PhD}$ degree in Computer Science Paris VI-P. and M. Curie University in 2013 on stereo 3D reconstruction taking into account bad weather conditions. From 2011, he is with the French institute of science and technology for transport, development and networks (IFSTTAR).

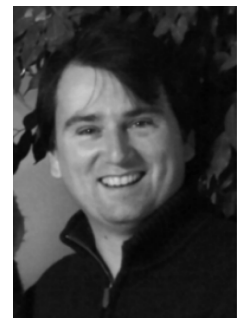

Jean-Philippe Tarel graduated from the Ecole Nationale des Ponts et Chaussées (ENPC), Paris, France (1991). He received his PhD degree in Applied Mathematics from Paris IX-Dauphine University in 1996. He was with the Institut National de Recherche en Informatique et Automatique (INRIA) from 1991 to 1996 and from 2001 to 2003. From 1997 to 1998 , he worked as a research associate at Brown University, USA. From 1999, he is a researcher in the French institute of science and technology for transport, development and networks (IFSTTAR and formerly LCPC), Paris, France. His research interests include $3 \mathrm{D}$ reconstruction, pattern recognition and detection.

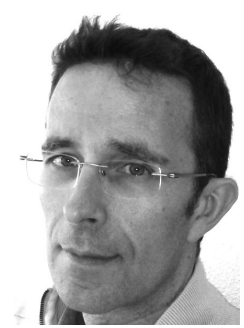

Pierre Charbonnier received the Ph.D. degree in electrical engineering from the University of NiceSophia Antipolis, in 1994. He did a post-doc at the Telecommunications and Remote Sensing Laboratory in Louvain-La-Neuve, Belgium in 1995-96. $\mathrm{He}$ is currently a Senior Researcher at the Cerema (center for expertise and engineering on risks, urban and country planning, environment and mobility), in Strasbourg, France. He leads the ERA 27 (CeremaIFSTTAR) research group. Since 2000, he is an external collaborator of the laboratory iCube (UMR 7357 CNRS-UDS). His research interests include inverse problems and regularization, classification and shape recognition (deformable models, robust estimation techniques) and computer vision. 